\title{
Analytical Techniques for a Numerical Solution of the Linear Volterra Integral Equation of the Second Kind
}

\author{
M. I. Berenguer, D. Gámez, A. I. Garralda-Guillem, \\ M. Ruiz Galán, and M. C. Serrano Pérez
}

E.U. Arquitectura Técnica, Departamento de Matemática Aplicada, Universidad de Granada, c/Severo Ochoa s/n, 18071 Granada, Spain

Correspondence should be addressed to M. Ruiz Galán, mruizg@ugr.es

Received 11 September 2009; Accepted 3 November 2009

Recommended by Viorel Barbu

In this work we use analytical tools-Schauder bases and Geometric Series theorem-in order to develop a new method for the numerical resolution of the linear Volterra integral equation of the second kind.

Copyright (C) 2009 M. I. Berenguer et al. This is an open access article distributed under the Creative Commons Attribution License, which permits unrestricted use, distribution, and reproduction in any medium, provided the original work is properly cited.

\section{Introduction}

Most mathematical models used in many problems of physics, biology, chemistry, engineering, and in other areas are based on integral equations like the linear Volterra integral equation of the second kind:

$$
x(t)=y_{0}(t)+\int_{a}^{t} k(t, s) x(s) d s \quad(t \in[a, b])
$$

where $k:[a, b] \times[a, b] \rightarrow \mathbb{R}$ and $y_{0}:[a, b] \rightarrow \mathbb{R}$ are two known continuous functions and $x:[a, b] \rightarrow \mathbb{R}$ is the unknown function to be determined.

Many authors have paid attention to the study of linear Volterra integral equation of the second kind from the viewpoint of their theoretical properties, numerical treatment, as well as its applications (see e.g., [1-4] and the references therein). Specifically, there are several numerical techniques to solve this equation such as the collocation method, finite element method, and spectral method. 
In this paper a new technique for solving this linear Volterra integral equation is shown. The method is based on two classical analytical tools: the Geometric Series theorem and Schauder bases in a Banach space. Schauder bases in adequate Banach spaces have been used in other numerical methods for solving some integral, differential, or integrodifferential equations (see [5-10]), although in each problem the analytical techniques are quite different, as fixed point theorems, duality mapping in a Banach space, and generalized least-squares methods.

Among the main advantages that our method presents over the classical ones, as collocation or quadrature (see [11]), we can point out that it is not necessary to solve linear equations systems. In addition, the involved integrals in our method are immediate and therefore, we do not have to use any quadrature method to calculate them.

The paper is organized as follows: some basic facts and properties on the Volterra equation (1.1) and on Schauder bases in $C([0,1])$ and $C\left([0,1]^{2}\right)$ are reviewed in Section 2. In Section 3 we define the approximating functions and we study the error. Finally, in Section 4 two numerical examples taken from $[12,13]$ are given to illustrate the theoretical results.

\section{Development of the Numerical Method: Classical Tools in Functional Analysis}

In this section we show some analytical techniques and some related results, useful for us in order to give our numerical method.

Let $C([a, b])$ be the Banach space of all continuous and real-valued functions on $[a, b]$, endowed with its usual sup-norm:

$$
\|f\|=\sup _{x \in[a, b]}|f(x)|, \quad(f \in C([a, b])) .
$$

Let us write

$$
L(C([a, b]))=\{T: C([a, b]) \longrightarrow C([a, b]): T \text { is linear and continuous }\},
$$

and let $T \in L(C([a, b]))$ be the operator defined by

$$
T x(t)=\int_{a}^{t} k(t, s) x(s) d s, \quad(a \leq t \leq b, x \in C([a, b])) .
$$

It can be shown by an induction argument and Fubini's theorem that

$$
\left(T^{n} x\right)(t)=\int_{a}^{t} \int_{a}^{t_{1}} \int_{a}^{t_{2}} \cdots \int_{a}^{t_{n-1}} k\left(t, t_{1}\right) k\left(t_{1}, t_{2}\right) \cdots k\left(t_{n-1}, t_{n}\right) x\left(t_{n}\right) d t_{n} d t_{n-1} \cdots d t_{1}
$$


for all $n \geq 1, x \in C([a, b])$ and $t \in[a, b]$ (we adopt the convention $\left.t_{0}=t\right)$. Hence,

$$
\begin{aligned}
\left|\left(T^{n} x\right)(t)\right| & \leq\|k\|^{n}\|x\| \int_{a}^{t} \int_{a}^{t_{1}} \int_{a}^{t_{2}} \cdots \int_{a}^{t_{n-1}} 1 d t_{n} \cdots d t_{1} \\
& =\|k\|^{n}\|x\| \frac{(t-a)^{n}}{n !} \leq\|k\|^{n}\|x\| \frac{(b-a)^{n}}{n !}
\end{aligned}
$$

and therefore,

$$
T^{n} \leq \frac{\|k\|^{n}(b-a)^{n}}{n !}
$$

where $\|k\|=\sup _{(x, y) \in[a, b]^{2}}|k(x, y)|$. The convergence of the series of real numbers $\sum_{n \geq 0}(\|k\|(b-a))^{n} / n$ ! ensures that the series $\sum_{n \geq 0} T^{n}$ is convergent in $L(C([a, b]))$.

This remark and the fact that (1.1) can be written equivalently as

$$
(I d-T) x=y_{0}
$$

lead us to consider the following result (see [14]).

Theorem 2.1 (geometric series theorem). Let $X$ be a Banach space and let $T: X \rightarrow X$ be a continuous and linear operator such that the series $\sum_{n \geq 0} T^{n}$ converges. Then, $I d-T$ is a continuous, linear, and bijective operator and $(I d-T)^{-1}=\sum_{n \geq 0} T^{n}$.

Then the unique solution $x$ of (2.7) is given by

$$
x=(I d-T)^{-1} y_{0}=\sum_{n \geq 0} T^{n} y_{0} .
$$

Thus, the sequence $\left\{y_{n}\right\}_{n \in \mathbb{N}}$ defined by $y_{n}=y_{0}+T y_{n-1}(n \geq 1)$ converges to the solution $x$ of (2.7).

By making use of an appropriate Schauder basis in the space $C\left([a, b]^{2}\right)$ we will replace each $T y_{j-1}(j \geq 1)$ for a new function $w_{j} \in C([a, b])$, easier to calculate, and in such a way the error $\left\|x-\left(y_{0}+w_{j}\right)\right\|$ is small enough.

Let us recall now that a sequence $\left\{b_{n}\right\}_{n \geq 1}$ in a Banach space $E$ is said to be a Schauder basis provided that for all $x \in E$ there exists a unique sequence of scalars $\left\{\beta_{n}\right\}_{n \geq 1}$ in such a way that

$$
x=\sum_{n \geq 1} \beta_{n} b_{n}
$$

The associated sequence of (continuous and linear) biorthogonal functionals $\left\{b_{n}^{*}\right\}_{n \geq 1}$ in the topological dual $E^{*}$ of $E$ is given by

$$
b_{n}^{*}\left(\sum_{k \geq 1} \beta_{k} b_{k}\right)=\beta_{n},
$$


and the sequence of (continuous and linear) projections $\left\{P_{n}\right\}_{n \geq 1}$ is defined by the partial sums:

$$
P_{n}\left(\sum_{k \geq 1} \beta_{k} b_{k}\right)=\sum_{k=1}^{n} \beta_{k} b_{k} .
$$

We now consider the usual Schauder basis for the space $C([0,1])$ (although the results in this paper work for equations stated over functions defined in $[a, b]$, we shall just consider the Banach space $C([0,1])$ for the sake of simplicity): for a dense sequence of distinct points $\left\{t_{i}\right\}_{i \geq 1}$ in $[0,1]$, with $t_{1}=0$ and $t_{2}=1$, we define

$$
b_{1}(t):=1, \quad(t \in[0,1])
$$

and for all $n \geq 2$ we stand $b_{n}$ for the piecewise linear continuous function determined by the points $\left\{t_{1}, \ldots, t_{n}\right\}$ satisfying

$$
\begin{gathered}
\forall k<n, \quad b_{n}\left(t_{k}\right)=0, \\
b_{n}\left(t_{n}\right)=1 .
\end{gathered}
$$

It is easy to obtain the sequence of biorthogonal functionals (see [15]): if $x \in C([0,1])$, then

$$
b_{1}^{*}(x)=x\left(t_{1}\right)
$$

and for all $n \geq 2$,

$$
b_{n}^{*}(x)=x\left(t_{n}\right)-\sum_{k=1}^{n-1} b_{k}^{*}(x) b_{k}\left(t_{n}\right) .
$$

In addition, the sequence of projections $\left\{P_{n}\right\}_{n \geq 1}$ satisfies the following interpolation property: for all $x \in C([0,1])$, for all $n \geq 1$, and for all $i \leq n$, we have that

$$
P_{n}(x)\left(t_{i}\right)=x\left(t_{i}\right)
$$

In what follows, for a real number $x,[x]$ will denote its integer part and $\sigma=\left(\sigma_{1}, \sigma_{2}\right)$ : $\mathbb{N} \rightarrow \mathbb{N} \times \mathbb{N}$ the bijective mapping defined by

$$
\sigma(n):= \begin{cases}(\sqrt{n}, \sqrt{n}), & \text { if }[\sqrt{n}]=\sqrt{n}, \\ \left(n-[\sqrt{n}]^{2},[\sqrt{n}]+1\right), & \text { if } 0<n-[\sqrt{n}]^{2} \leq[\sqrt{n}], \\ \left([\sqrt{n}]+1, n-[\sqrt{n}]^{2}-[\sqrt{n}]\right), & \text { if }[\sqrt{n}]<n-[\sqrt{n}]^{2} .\end{cases}
$$

If $\left\{b_{i}\right\}_{i \geq 1}$ and $\left\{\widehat{b}_{j}\right\}_{j \geq 1}$ are Schauder bases for the space $C([0,1])$, then the sequence

$$
B_{n}(s, t):=b_{i}(s) \widehat{b}_{j}(t), \quad(s, t \in[0,1])
$$


with $\sigma(n)=(i, j)$ is a Schauder basis for $C\left([0,1]^{2}\right)$ (the proof of this fact can be found in $[15,16])$. Therefore, from now on, if $\left\{t_{i}: i \geq 1\right\}$ is a dense subset of distinct points in $[0,1]$, with $t_{1}=0$ and $t_{2}=1$, and $\left\{b_{i}\right\}_{i \geq 1}$ is the associated usual Schauder basis, then we will write $\left\{B_{n}\right\}_{n \geq 1}$ to denote the Schauder basis for $C\left([0,1]^{2}\right)$ obtained in this "natural" way. It is not difficult to check that this basis satisfies similar properties to the ones for the one-dimensional case: for all $s, t \in[0,1]$,

$$
B_{1}(s, t)=1
$$

and for $n \geq 2$

$$
B_{n}\left(t_{i}, t_{j}\right)= \begin{cases}1, & \text { if } \sigma(n)=(i, j) \\ 0, & \text { if } \sigma^{-1}(i, j)<n\end{cases}
$$

In particular, the sequence of biorthogonal functionals $\left\{B_{n}^{*}\right\}_{n \geq 1}$ can be easily obtained: if $x \in$ $C\left([0,1]^{2}\right)$, then

$$
B_{1}^{*}(x)=x\left(t_{1}, t_{1}\right)
$$

and for all $n \geq 2$, if $\sigma(n)=(i, j)$, we have

$$
B_{n}^{*}(x)=x\left(t_{i}, t_{j}\right)-\sum_{k=1}^{n-1} B_{k}^{*}(x) B_{k}\left(t_{i}, t_{j}\right)
$$

As a consequence, the sequence of projections $\left\{Q_{n}\right\}_{n \geq 1}$ satisfies

$$
Q_{n}(x)\left(t_{i}, t_{j}\right)=x\left(t_{i}, t_{j}\right)
$$

whenever $n, i, j \in \mathbb{N}$ and $\sigma^{-1}(i, j) \leq n$.

Under some weak condition, we can estimate the rate of the convergence of the sequence of projections in the bidimensional case. To this purpose, consider the dense subset $\left\{t_{i}\right\}_{i \geq 1}$ of distinct points in $[0,1]$ and let $T_{n}$ be the set $\left\{t_{1}, \ldots, t_{n}\right\}$ ordered in an increasing way for $n \geq 2$. Let $\Delta T_{n}$ denotes the maximum distance between two consecutive points of $T_{n}$.

The following result is derived easily from (2.23) and the Mean-Value theorem.

Proposition 2.2. Let $x \in C^{1}\left([0,1]^{2}\right)$ and write

$$
M:=\max \left\{\left\|\frac{\partial x}{\partial s}\right\|,\left\|\frac{\partial x}{\partial t}\right\|\right\} .
$$

If $n \geq 2$, then

$$
\left\|x-Q_{n^{2}}(x)\right\| \leq 4 M \Delta T_{n}
$$




\section{Numerical Study of the Linear Volterra Integral Equation of the Second Kind: Convergence and Error}

We are now in a position to define the functions $w_{j} \in C([0,1])$ announced in the preceding section. Let $m \in \mathbb{N}, w_{0}=0$ and, with the notation above, define inductively, for $j \in\{1, \ldots, m\}$, the functions

$$
w_{j}(t)=\int_{0}^{t} Q_{n^{2}}\left(k(t, s)\left(y_{0}(s)+w_{j-1}(s)\right)\right) d s,
$$

where $n_{j}$ (for $j \in\{1, \ldots, m\}$ ) are natural numbers.

We obtain a first estimation of the error $\left\|x-\left(y_{0}+w_{m}\right)\right\|$.

Proposition 3.1. Maintaining the notation,

$$
\left\|x-\left(y_{0}+w_{m}\right)\right\| \leq\left\|T^{m+1}\right\|\left\|(I d-T)^{-1}\right\|\left\|y_{0}\right\|+\sum_{n=0}^{m-1}\|T\|^{m-1-n}\left\|T\left(y_{0}+w_{n}\right)-w_{n+1}\right\| .
$$

Proof. The triangle inequality gives

$$
\left\|x-\left(y_{0}+w_{m}\right)\right\| \leq\left\|x-y_{m}\right\|+\left\|y_{m}-\left(y_{0}+w_{m}\right)\right\| .
$$

For the first summand we have

$$
\begin{aligned}
\left\|x-y_{m}\right\| & =\left\|\sum_{n \geq 0} T^{n} y_{0}-\sum_{n \geq 0}^{m} T^{n} y_{0}\right\| \\
& =\left\|\sum_{n \geq m+1} T^{n} y_{0}\right\| \\
& \leq\left\|T^{m+1}\right\|\left\|\sum_{n \geq 0} T^{n} y_{0}\right\| \\
& =\left\|T^{m+1}\right\|\left\|(I d-T)^{-1} y_{0}\right\| \\
& \leq\left\|T^{m+1}\right\|\left\|(I d-T)^{-1}\right\|\left\|y_{0}\right\| .
\end{aligned}
$$

For the second one, by an induction argument we can show that

$$
\left\|y_{m}-\left(y_{0}+w_{m}\right)\right\| \leq \sum_{n=0}^{m-1}\|T\|^{m-1-n}\left\|T\left(y_{0}+w_{n}\right)-w_{n+1}\right\|
$$


Indeed, for $m=1$, the result is clearly true. Suppose that it holds for $p \leq m-1$. Then,

$$
\begin{aligned}
\left\|y_{m}-\left(y_{0}+w_{m}\right)\right\| & =\left\|\left(y_{0}+T y_{m-1}\right)-\left(y_{0}+w_{m}\right)\right\| \\
& =\left\|T y_{m-1}-w_{m}\right\| \\
& \leq\left\|T y_{m-1}-T\left(y_{0}+w_{m-1}\right)\right\|+\left\|T\left(y_{0}+w_{m-1}\right)-w_{m}\right\| \\
& \leq\|T\|\left\|y_{m-1}-\left(y_{0}+w_{m-1}\right)\right\|+\left\|T\left(y_{0}+w_{m-1}\right)-w_{m}\right\| \\
& \leq\|T\|\left(\sum_{n=0}^{m-2}\|T\|^{(m-1)-1-n}\left\|T\left(y_{0}+w_{n}\right)-w_{n+1}\right\|\right)+\left\|T\left(y_{0}+w_{m-1}\right)-w_{m}\right\| \\
& =\sum_{n=0}^{m-1}\|T\|^{m-1-n}\left\|T\left(y_{0}+w_{n}\right)-w_{n+1}\right\| .
\end{aligned}
$$

In order to control the sum in the right-hand term of the inequality stated in Proposition 3.1, let us assume that $k, y_{0} \in C^{1}\left([0,1]^{2}\right)$ and let us write

$$
h:=\max _{j=1, \ldots, m} \Delta T_{n_{j}}
$$

Then we derive from Proposition 2.2 that

$$
\begin{aligned}
\left|\left(T\left(y_{0}+w_{j}\right)-w_{j+1}\right)(t)\right| & =\left|\int_{0}^{t}\left(k(t, s)\left(y_{0}(s)+w_{j}(s)\right)\right)-Q_{n_{j+1}}\left(k(t, s)\left(y_{0}(s)+w_{j}(s)\right)\right) d s\right| \\
& \leq 4 M_{j} h,
\end{aligned}
$$

where

$$
M_{j}=\max \left\{\left\|\frac{\partial\left(k\left(y_{0}+w_{j}\right)\right)}{\partial s}\right\|,\left\|\frac{\partial\left(k\left(y_{0}+w_{j}\right)\right)}{\partial t}\right\|\right\}
$$

and hence,

$$
\left\|T\left(y_{0}+w_{j}\right)-w_{j+1}\right\| \leq 4 M_{j} h
$$

To arrive at the announced estimation we finally have the following.

Proposition 3.2. The sequences $\left\{\left\|w_{j}\right\|\right\}_{j \in \mathbb{N}}$ and $\left\{\left\|w_{j}^{\prime}\right\|\right\}_{j \in \mathbb{N}}$ are bounded and, as a consequence, the sequence $\left\{M_{j}\right\}_{j \in \mathbb{N}}$ is also bounded. 
Proof. First we show that for all $t \in[0,1]$,

$$
\left|w_{j}(t)\right| \leq\left\|y_{0}\right\|\left(\sum_{n=1}^{j} \frac{t^{n}}{n !}\|k\|^{n}\right),
$$

and, as a consequence,

$$
\left\|w_{j}\right\| \leq\left\|y_{0}\right\|\left(\sum_{n=1}^{j} \frac{\|k\|^{n}}{n !}\right) \leq\left\|y_{0}\right\| e^{\|k\|},
$$

by using an inductive argument: since the Schauder basis $\left\{B_{n}\right\}_{n \geq 1}$ is clearly monotone (normone projections), we have

$$
\left|w_{1}(t)\right| \leq \int_{0}^{t}\|k\|\left\|y_{0}\right\| d s \leq\left\|y_{0}\right\|\|k\| t
$$

Suppose that the result holds for $p \leq j-1$. Then

$$
\begin{aligned}
\left|w_{j}(t)\right| & \leq \int_{0}^{t}\|k\|\left\|y_{0}\right\| d s+\int_{0}^{t}\|k\|\left|w_{j-1}(s)\right| d s \\
& \leq\left\|y_{0}\right\|\|k\| t+\|k\|\left\|y_{0}\right\| \int_{0}^{t}\left(\sum_{n=1}^{j-1} \frac{s^{n}}{n !}\|k\|^{n}\right) d s \\
& \leq\left\|y_{0}\right\|\|k\| t+\left\|y_{0}\right\|\left(\sum_{n=1}^{j-1} \frac{t^{n+1}}{(n+1) !}\|k\|^{n+1}\right) \\
& \leq\left\|y_{0}\right\|\left(\sum_{n=1}^{j} \frac{t^{n}}{n !}\|k\|^{n}\right) .
\end{aligned}
$$

On the other hand,

$$
w_{j}^{\prime}(t)=Q_{n_{j}{ }^{2}}\left(k(t, t)\left(y_{0}(t)+w_{j-1}(t)\right)\right)
$$

and thus

$$
\left\|w_{j}^{\prime}\right\| \leq\|k\|\left(\left\|y_{0}\right\|+\left\|w_{j-1}\right\|\right)
$$


Finally, the bounding of $\left\{M_{j}\right\}_{j \in \mathbb{N}}$ follows from

$$
\begin{gathered}
\left\|\frac{\partial\left(k\left(y_{0}+w_{j}\right)\right)}{\partial t}\right\| \leq\left\|\frac{\partial k}{\partial t}\right\|\left(\left\|y_{0}\right\|+\left\|w_{j}\right\|\right), \\
\left\|\frac{\partial\left(k\left(y_{0}+w_{j}\right)\right)}{\partial s}\right\| \leq\left\|\frac{\partial k}{\partial s}\right\|\left(\left\|y_{0}\right\|+\left\|w_{j}\right\|\right)+\|k\|\left(\left\|y_{0}^{\prime}\right\|+\left\|w_{j}^{\prime}\right\|\right) .
\end{gathered}
$$

Letting $\beta=\sup \left\{M_{j}\right\}_{j \in \mathbb{N}}$ and $\delta=4 \beta \sum_{j \geq 0}\|T\|^{j}$, from (3.10) we have

$$
\sum_{j=0}^{m-1}\|T\|^{m-1-j}\left\|T\left(y_{0}+w_{j}\right)-w_{j+1}\right\| \leq \delta h
$$

Then Propositions 3.1 and 3.2 imply the following result, which gives an upper bound of the error.

Theorem 3.3. Suppose $k, y_{0} \in C^{1}\left([0,1]^{2}\right)$ and let $\left\{w_{0}, w_{1}, \ldots, w_{m}\right\}$ be the functions defined in (3.1). Then, there exists $\delta>0$ such that

$$
\left\|x-\left(y_{0}+w_{m}\right)\right\| \leq\left\|T^{m+1}\right\|\left\|(I d-T)^{-1}\right\|\left\|y_{0}\right\|+\delta h .
$$

\section{Numerical Examples}

The behaviour of this method is illustrated by means of the following two examples. The computations associated with the numerical experiments were carried out using Mathematica 7.

The chosen dense subset of $[0,1]$ is

$$
\left\{0,1, \frac{1}{2}, \frac{1}{4}, \frac{3}{4}, \ldots, \frac{1}{2^{k}}, \frac{3}{2^{k}}, \ldots, \frac{2^{k}-1}{2^{k}}, \ldots\right\} .
$$

To construct the functions $w_{j}$ introduced in Section 3, we have taken $n_{j}=n$ in the expression (3.1). For such a choice the value of $h$ in Section 3 is $1 /(n-1)$.

In both cases we exhibit, for $n=9,17,33$, and 65 the absolute errors committed in eight representative points of $[0,1]$ when we approximate the exact solution $x$ by the function $y_{0}+w_{m}$, where $m$ has been determined in the following way: for each $j \in \mathbb{N}$ we note

$$
E_{n, j}=\max _{i=1, \ldots, n}\left|\left(y_{0}+w_{j}\right)\left(t_{i}\right)-x\left(t_{i}\right)\right|
$$

and choose $m$ satisfying

$$
\frac{E_{n, m}}{E_{n, m+1}}<1+10^{-2}
$$


Table 1: Numerical results for Example 4.1.

\begin{tabular}{lcccc}
\hline$t$ & $n=9, m=2$ & $n=17, m=2$ & $n=33, m=2$ & $n=65, m=3$ \\
\hline 0.125 & $2.13 \times 10^{-8}$ & $6.85 \times 10^{-9}$ & $1.82 \times 10^{-9}$ & $4.63 \times 10^{-10}$ \\
0.250 & $1.76 \times 10^{-6}$ & $4.68 \times 10^{-7}$ & $1.19 \times 10^{-7}$ & $2.98 \times 10^{-8}$ \\
0.375 & $2.11 \times 10^{-5}$ & $5.41 \times 10^{-6}$ & $1.36 \times 10^{-6}$ & $3.41 \times 10^{-7}$ \\
0.500 & $1.21 \times 10^{-4}$ & $3.07 \times 10^{-5}$ & $7.66 \times 10^{-6}$ & $1.93 \times 10^{-6}$ \\
0.625 & $4.73 \times 10^{-4}$ & $1.17 \times 10^{-4}$ & $2.83 \times 10^{-5}$ & $7.47 \times 10^{-6}$ \\
0.750 & $1.42 \times 10^{-3}$ & $3.43 \times 10^{-4}$ & $7.11 \times 10^{-5}$ & $2.23 \times 10^{-5}$ \\
0.875 & $3.55 \times 10^{-3}$ & $7.61 \times 10^{-4}$ & $6.32 \times 10^{-5}$ & $5.24 \times 10^{-5}$ \\
1 & $7.32 \times 10^{-3}$ & $1.00 \times 10^{-3}$ & $5.73 \times 10^{-4}$ & $6.92 \times 10^{-5}$ \\
\hline
\end{tabular}

Table 2: Compared results.

\begin{tabular}{lccc}
\hline$h$ & $n$ & $\|x-u\|_{h, 2}$ & $\left\|x-\left(y_{0}+w_{m}\right)\right\|_{h, 2}$ \\
\hline $1 / 8$ & 9 & $1.16 \times 10^{-1}$ & $3.43 \times 10^{-3}$ \\
$1 / 16$ & 17 & $5.51 \times 10^{-2}$ & $7.12 \times 10^{-4}$ \\
$1 / 32$ & 33 & $2.68 \times 10^{-2}$ & $1.52 \times 10^{-4}$ \\
$1 / 64$ & 65 & $1.32 \times 10^{-2}$ & $2.81 \times 10^{-5}$ \\
\hline
\end{tabular}

Example 4.1. The equation (see [12]),

$$
x(t)=t^{5}-\frac{t^{8}}{7}+\int_{0}^{t} s t x(s) d s, \quad(t \in[0,1])
$$

has exact solution $x(t)=t^{5}$.

In Table 2 we compare the approximations $y_{0}+w_{m}$ obtained as described above with the collocation solution $u$ shown in [12] in terms of the norm

$$
\|v\|_{h, 2}:=\left(h \sum_{k=1}^{n} v\left(t_{k}\right)^{2}\right)^{1 / 2}
$$

introduced in the cited article. We point out that $h=1 /(n-1)$ in both methods.

Example 4.2. Second example is taken from [13]. We consider the equation

$$
x(t)=1+\int_{0}^{t}(s-t) x(s) d s, \quad(t \in[0,1]),
$$

whose exact solution is $x(t)=\cos (t)$. 
Table 3: Numerical results for Example 4.2.

\begin{tabular}{lcccc}
\hline$t$ & $n=9, m=2$ & $n=17, m=2$ & $n=33, m=2$ & $n=65, m=2$ \\
\hline 0.1 & $1.21 \times 10^{-5}$ & $2.14 \times 10^{-6}$ & $3.66 \times 10^{-7}$ & $9.97 \times 10^{-8}$ \\
0.2 & $3.42 \times 10^{-5}$ & $5.83 \times 10^{-6}$ & $1.58 \times 10^{-6}$ & $4.16 \times 10^{-7}$ \\
0.3 & $5.82 \times 10^{-5}$ & $1.57 \times 10^{-5}$ & $3.67 \times 10^{-6}$ & $8.77 \times 10^{-7}$ \\
0.4 & $8.95 \times 10^{-5}$ & $2.46 \times 10^{-5}$ & $6.48 \times 10^{-6}$ & $1.58 \times 10^{-6}$ \\
0.5 & $1.45 \times 10^{-4}$ & $3.27 \times 10^{-5}$ & $6.35 \times 10^{-6}$ & $6.76 \times 10^{-7}$ \\
0.6 & $2.39 \times 10^{-4}$ & $5.49 \times 10^{-5}$ & $1.31 \times 10^{-5}$ & $3.35 \times 10^{-6}$ \\
0.7 & $2.94 \times 10^{-4}$ & $6.75 \times 10^{-5}$ & $1.76 \times 10^{-5}$ & $4.48 \times 10^{-6}$ \\
0.8 & $3.52 \times 10^{-4}$ & $9.30 \times 10^{-5}$ & $2.24 \times 10^{-5}$ & $5.52 \times 10^{-6}$ \\
0.9 & $3.95 \times 10^{-5}$ & $1.07 \times 10^{-4}$ & $2.76 \times 10^{-5}$ & $6.80 \times 10^{-6}$ \\
1 & $1.50 \times 10^{-3}$ & $9.24 \times 10^{-4}$ & $5.02 \times 10^{-4}$ & $2.61 \times 10^{-4}$ \\
\hline
\end{tabular}

The computed results by the suggested method for $n=9(h=1 / 8)$ improve the obtained ones in [13] for $h=1 / 10$.

\section{Acknowledgments}

This Research is Partially supported by M.E.C. (Spain) and FEDER project no. MTM200612533, and by Junta de Andalcía Grant FQM359.

\section{References}

[1] C. T. H. Baker, The Numerical Treatment of Integral Equations, Monographs on Numerical Analysis, Clarendon Press, Oxford, UK, 1977.

[2] C. T. H. Baker, "A perspective on the numerical treatment of Volterra equations," Journal of Computational and Applied Mathematics, vol. 125, no. 1-2, pp. 217-249, 2000.

[3] L. M. Delves and J. L. Mohamed, Computational Methods for Integral Equations, Cambridge University Press, Cambridge, UK, 1985.

[4] Z. Wan, Y. Chen, and Y. Huang, "Legendre spectral Galerkin method for second-kind Volterra integral equations," Frontiers of Mathematics in China, vol. 4, no. 1, pp. 181-193, 2009.

[5] M. I. Berenguer, M. A. Fortes, A. I. Garralda Guillem, and M. Ruiz Galán, "Linear Volterra integrodifferential equation and Schauder bases," Applied Mathematics and Computation, vol. 159, no. 2, pp. 495-507, 2004.

[6] E. Castro, D. Gámez, A. I. Garralda Guillem, and M. Ruiz Galán, "High order linear initial-value problems and Schauder bases," Applied Mathematical Modelling, vol. 31, no. 12, pp. 2629-2638, 2007.

[7] D. Gámez, A. I. Garralda Guillem, and M. Ruiz Galán, "High-order nonlinear initial-value problems countably determined," Journal of Computational and Applied Mathematics, vol. 228, no. 1, pp. 77-82, 2009.

[8] D. Gámez, A. I. Garralda Guillem, and M. Ruiz Galán, "Nonlinear initial-value problems and Schauder bases," Nonlinear Analysis: Theory, Methods \& Applications, vol. 63, no. 1, pp. 97-105, 2005.

[9] A. Palomares, M. Pasadas, V. Ramírez, and M. Ruiz Galán, "A convergence result for a least-squares method using Schauder bases," Mathematics and Computers in Simulation, vol. 77, no. 2-3, pp. 274-281, 2008.

[10] A. Palomares and M. Ruiz Galán, "Isomorphisms, Schauder bases in Banach spaces, and numerical solution of integral and differential equations," Numerical Functional Analysis and Optimization, vol. 26, no. 1, pp. 129-137, 2005.

[11] H. Brunner and P. J. van der Houwen, The Numerical Solution of Volterra Equations, vol. 3 of CWI Monographs, North-Holland, Amsterdam, The Netherlands, 1986.

[12] H. Brunner, L. Qun, and Y. Ningning, "The iterative correction method for Volterra integral equations," BIT. Numerical Mathematics, vol. 36, no. 2, pp. 221-228, 1996. 
[13] K. Maleknejad and N. Aghazadeh, "Numerical solution of Volterra integral equations of the second kind with convolution kernel by using Taylor-series expansion method," Applied Mathematics and Computation, vol. 161, no. 3, pp. 915-922, 2005.

[14] K. Atkinson and W. Han, Theoretical Numerical Analysis: A Functional Analysis Framework, vol. 39 of Texts in Applied Mathematics, Springer, Dordrecht, The Netherlands, 3rd edition, 2009.

[15] Z. Semadeni, "Product Schauder bases and approximation with nodes in spaces of continuous functions," Bulletin de l'Académie Polonaise des Sciences, vol. 11, pp. 387-391, 1963.

[16] B. R. Gelbaum and J. Gil de Lamadrid, "Bases of tensor products of Banach spaces," Pacific Journal of Mathematics, vol. 11, pp. 1281-1286, 1961. 


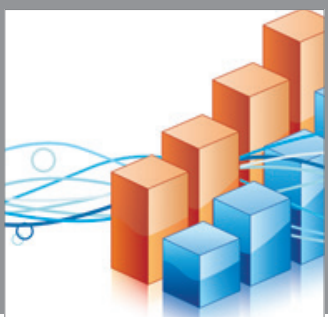

Advances in

Operations Research

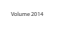

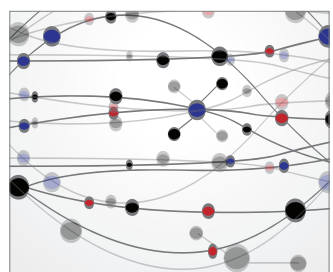

\section{The Scientific} World Journal
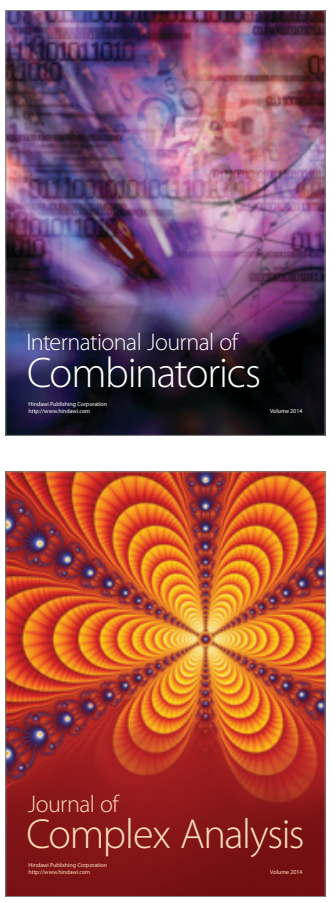

International Journal of

Mathematics and

Mathematical

Sciences
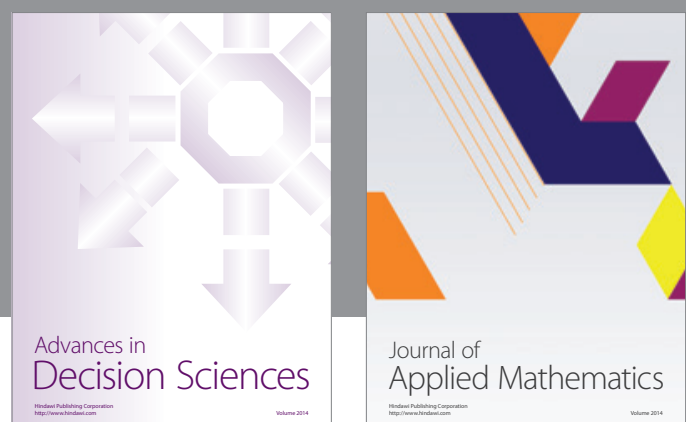

Journal of

Applied Mathematics
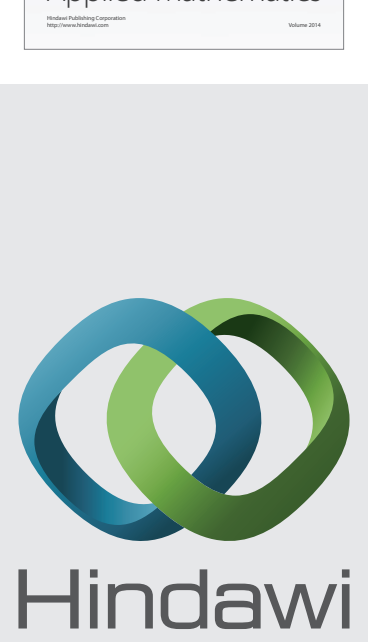

Submit your manuscripts at http://www.hindawi.com
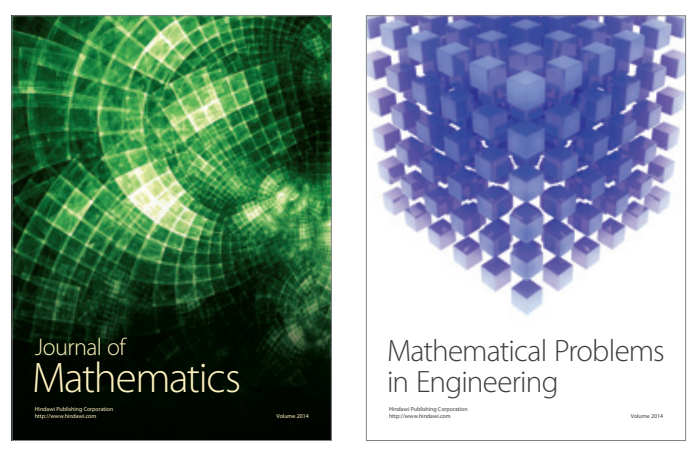

Mathematical Problems in Engineering
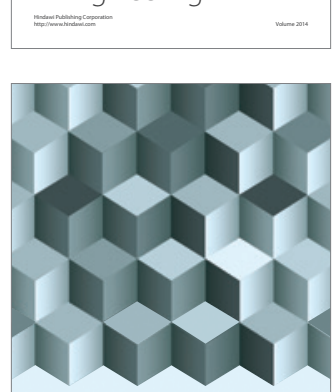

Journal of

Function Spaces
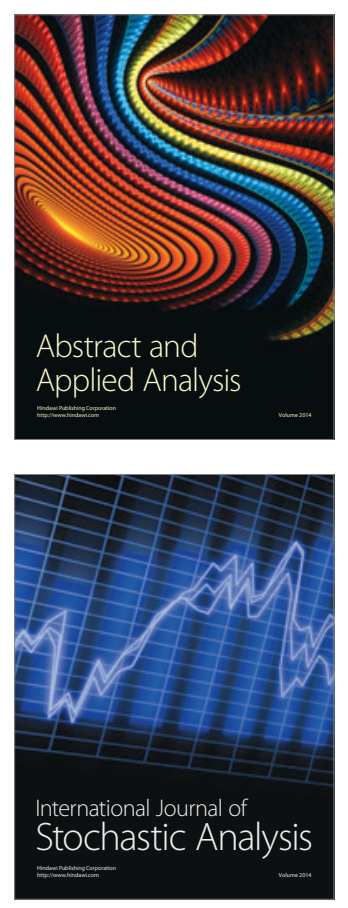

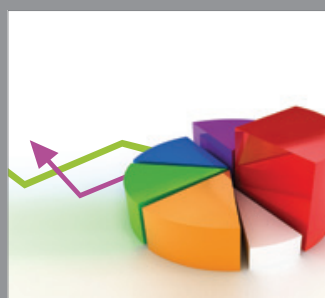

ournal of

Probability and Statistics

Promensencen
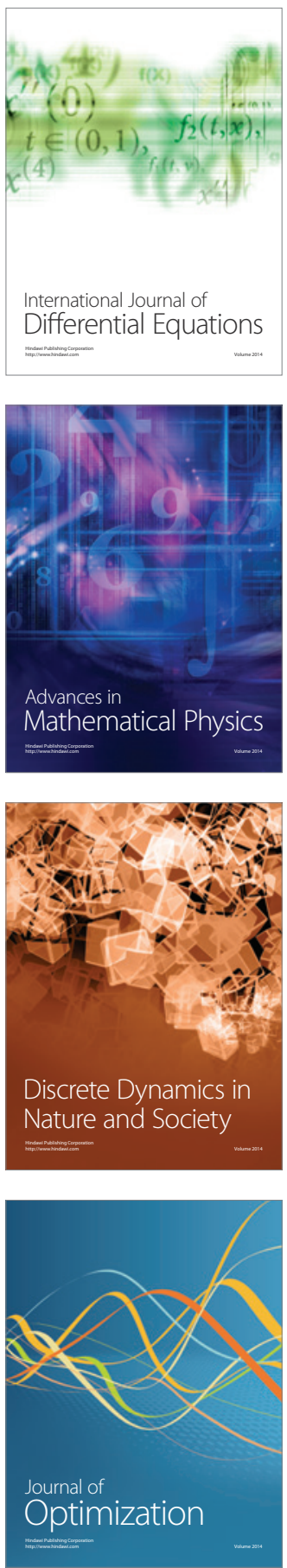